\title{
Regard complémentaire - Sociologie et didactique. Vers un espace commun de problématisation
}

Sociology and didactics. Towards a common space of problematisation

Patrick Rayou

\section{OpenEdition}

Journals

Édition électronique

URL : http://journals.openedition.org/educationdidactique/1905

DOI : 10.4000/educationdidactique.1905

ISSN : 2111-4838

Éditeur

Presses universitaires de Rennes

Édition imprimée

Date de publication : 30 juillet 2014

Pagination : 91-100

ISSN : 1956-3485

\section{Référence électronique}

Patrick Rayou, «Regard complémentaire - Sociologie et didactique. Vers un espace commun de problématisation », Éducation et didactique [En ligne], 8-1 | 2014, mis en ligne le 15 septembre 2016, consulté le 01 mai 2019. URL : http://journals.openedition.org/educationdidactique/1905; DOI : 10.4000/educationdidactique. 1905 
SOCIOLOGIE ET DIDACTIQUE. VERS UN ESPACE COMMUN DE PROBLÉMATISATION

Patrick Rayou

Université Paris 8, Circeft Escol 
Cette riche journée d'étude ${ }^{1}$ a montré des volontés de dialogue, sinon d'unification, entre des approches diverses et parfois contradictoires de la didactique. Des argumentaires forts y ont été développés de part et d'autre, qui suscitent souvent chez qui les écoute, le sentiment étrange d'être d'accord avec chacun... La nécessité s'impose alors de saisir le domaine de validité de chacune des prises de parti dans le débat sur la généricité ou la spécificité de la ou des didactique(s) en faisant l'hypothèse qu'elles se complètent au lieu de ne viser qu'à se substituer les unes aux autres. Il s'agit donc de comprendre ce qui constitue ces tensions en interrogeant, comme l'ont fait les débats, la notion même de " discipline " auxquelles, d'une manière ou d'une autre toutes les approches en termes de didactique sont adossées, puis de proposer quelques pistes susceptibles, d'un point de vue sociologique, de contribuer au développement d'un espace commun de problématisation entre sociologues et didacticiens.

\section{UNE UNIFICATION NÉCESSAIRE ET COMPLEXE}

À travers les points de vue développés dans ce dossier, la tension entre pluriel et singulier de la/des didactique(s) semble tout simplement constitutive de ce champ de recherche. Comme le souligne Bernard Schneuwly, les «subject didactics» peuvent être considérés comme un champ qui décrit et explique les conditions d'enseignement et d'apprentissage dans un domaine donné, mais aussi comme le développement d'une recherche fondamentale sur ces conditions. Avec d'autres termes, Yves Chevallard souligne le nécessaire passage d'une didactique des disciplines scolaires à une didactique conçue comme science anthropologique. Pourquoi une telle tension entre spécificité et généricité, somme toute constitutive et motrice de toute approche scientifique, est-elle si difficile à admettre en matière de didactique? Sans doute du fait d'une difficile institutionnalisation dans le champ académique, compliquée par l'opposition, en France, entre polyvalence de l'enseignement dans le premier degré et spécialisation dans le second, qui fait se poser de façon très vive la question de savoir à qui elle appartient. Le risque d'une didactique « hors-sol » serait bien évidemment de développer un discours général rompant avec les caractéristiques des apprentissages. Le risque inverse serait celui de la fragmentation de communautés de didacticiens qui réserverait le débat scientifique aux « indigènes de la discipline» (Chevallard, dans ce numéro).

Une issue à ce qui pourrait être un dilemme est proposée par Helmut Johannes Vollmer: cette issue envisage la possible théorisation des didactiques disciplinaires particulières par une didactique disciplinaire générale (ou généralisée) qui les contiendrait partiellement. Elle suggère ainsi que l'ambiguité du statut généraliste ou spécialiste de la didactique renvoie elle-même à ce qu'est une discipline et que les apports de cette journée peuvent aider à élucider.

\section{UNE DIALECTIQUE DES DISCIPLINES}

\section{Discipline universitaire et discipline enseignée}

Il s'agit de commencer par se demander de quoi on parle quand on parle de discipline. Pour plus de clarté, Bernard Schneuwly parle de « subjects didactics ", car le français ne distingue pas entre « discipline » au sens universitaire, et « discipline » au sens scolaire de discipline enseignée. L'allemand utilise lui aussi un terme unique "Fach » pour caractériser ces deux pratiques sociales différentes. Ne pas faire la distinction fait courir le risque d'importer dans la didactique comme recherche les fragmentations des disciplines scolairement distribuées. Or, comme le précise Yves Chevallard, la position de chercheur en didactique suppose une rupture épistémologique avec la position de l'enseignant dont elle constitue, sans adopter pour autant une position de surplomb, l'univers professionnel en objet d'étude.

\section{Généricité et spécificité de la discipline}

Une autre complexité, déjà croisée, est celle de la cohabitation, au sein même de la didactique de caractéristiques plus ou moins générales. Comme le l'a déjà résumé Jean-Louis Martinand, une didactique indépendante ne serait plus une didactique de discipline et une didactique où l'amalgame entre didacticiens de diverses origines ne se produirait pas serait vraisemblablement stérile (Martinand, 2006). Pour dépasser ce dilemme, Yves Chevallard propose de considérer que la didactique est d'abord la science $d u$ didactique présent dans un continuum qui va du générique de l'humanité au spécifique des systèmes didactiques, en passant par les civili- 
Patrick Rayou

sations, les sociétés, les écoles, les pédagogies et les disciplines proprement dites. Ce qu'une institution nous présente comme discipline n'en est donc pas la substance et le passage par le fondamental de la discipline nous aide à accéder au spécialisé. Une telle dialectique autorise et légitime alors un projet de didactique comparée, dont la construction de cadres conceptuels n'est pas simple, mais qu'appelle, selon Jean-Louis Martinand, le renouvellement de l'étude de la professionnalité enseignante.

\section{Disciplines et contingences}

Si les disciplines d'enseignement se disent simultanément au singulier et au pluriel, elles doivent aussi s'appréhender dans une contingence lisible dans les négociations constantes dont elles font l'objet et qui les font évoluer, y compris sous l'influence de la didactique, selon Jean-Louis Martinand. Forquin (2008) a proposé, pour rendre compte de ce phénomène, de distinguer, du plus essentiel au plus contingent, entre forme scolaire (Vincent, Lahire \& Thin, 1994), forme curriculaire et forme disciplinaire. La première est un ensemble cohérent de traits caractéristiques de l'école. La seconde un dispositif d'enseignement, où se rencontrent une dimension « systémique » articulant des composantes de savoirs correspondant ou non à des "matières" ou à des « disciplines», et une dimension "séquentielle » qui programme dans le temps l'enseignement de chacune de ces composantes selon un plan déterminé. La forme disciplinaire suppose, quant à elle, un plus grand nombre d'exigences, comme le recrutement d'un corps enseignant spécialisé ou l'existence d'un corpus homogène de savoirs de référence. JeanClaude Forquin reprend ainsi la distinction établie par Martinand (2001) entre matrices disciplinaires et matrices curriculaires, susceptible d'expliquer par exemple qu'un véritable curriculum d'éducation technologique aurait pu être mis en place au collège sans passer par la constitution d'une discipline séparée ou que des «enseignements à » peuvent exister sans correspondre à des disciplines établies.

\section{Disciplines et co-construction}

Une autre manière de dialectiser les disciplines est de montrer que leur existence réelle dérive certes de leur définition institutionnelle, mais aussi de la façon dont elles sont reconstruites par les acteurs, les élèves notamment. C'est ce qu'a proposé Reuter (2007) avec la notion de "conscience disciplinaire " (voir aussi Reuter, dans ce numéro), qui s'intéresse à la mise en œuvre des disciplines. Celle-ci suppose de fait une reconstruction à laquelle les élèves participent largement, en percevant par exemple comme plus ou moins homogènes le Français, les Mathématiques ou l'Éducation Physique et Sportive, ou en appréhendant différentiellement leurs commencements dans telle ou telle discipline. Celles-ci sont, par-delà leurs caractéristiques intrinsèques naturalisées, des espaces vécus qu'il y a lieu de prendre en compte, si l'on veut comprendre les attitudes des élèves en matière d'incivilités ou de décrochages.

Ces différents modes de dialectisation des disciplines appellent des approches contextualisantes auxquelles la sociologie peut, elle-même, apporter son concours.

\section{UNE COLLABORATION ENTRE SOCIOLOGIE ET DIDACTIQUE}

\section{La sociologie et les transmissions culturelles}

\section{Généricité et spécificité}

La constitution d'un espace commun de problématisation nécessaire à la compréhension de ce que sont les disciplines scolaires est rendue nécessaire par le fait que la culture scolaire se caractérise, comme le souligne Forquin (2008), non seulement par des « contenus», mais par des mises en forme de ceux-ci «à travers notamment l'élaboration de programmes d'études obéissant à une forte codification didactique » (p. 17).

Jean-Claude Forquin esquisse à grands traits trois apports, selon lui majeurs, qui peuvent rendre compte de la double nature, générique et spécifique, des disciplines. Celui qui met l'accent sur la nécessité d'opérer une transposition entre la forme savante et la forme scolaire des savoirs (Chevallard, 1985/1991), celui qui insiste sur l'importance des pratiques sociales qui entrent également dans les apprentissages scolaires (Martinand, 1986), celui qui montre dans l'école, plus qu'une instance de traitement des savoirs disponibles, un creuset où s'élaborent des connaissances, des postures, des dispositions consti- 
tuant le noyau d'une culture scolaire sui generis et donc spécifique (Chervel, 1977, 1998). Forquin (2008) souligne alors «l'intérêt ou la nécessité d'une confrontation et d'un dialogue approfondis entre sociologie du curriculum, sociologie de la culture, didactique et histoire de l'éducation. » (p. 17).

Si la sociologie de l'éducation peut contribuer à la compréhension de cette dialectique entre générique et spécifique, c'est parce qu'elle est, au moins depuis les travaux d'Émile Durkheim, consciente de ce que le processus général de transmission de normes et valeurs d'une génération à l'autre se décline toujours en fonction de l'ensemble des autres institutions sociales et de leurs évolutions. Pour l'auteur de L'Évolution pédagogique en France (Durkheim, 1990/1938) les enfants sont ainsi simultanément intégrés dans la société politique en général et dans un milieu spécial qui requiert des connaissances et compétences particulières. Les activités sociales auxquelles préparaient, par la dialectique et la dispute, l'enseignement médiéval, se trouvèrent ainsi, par exemple, historiquement dépassées par l'essor de la bourgeoisie et le raisonnement expérimental prit la place de la logique comme fondement des programmes éducatifs. Durkheim fut, lui-même, le témoin de transformations de l'enseignement du second degré qui manifestaient, selon lui, de manière exacerbée et préoccupante, les tensions entre formation générale et formations spécifiques sur fond de perte de crédit de l'explication religieuse du monde.

\section{Une sociologie du curriculum}

Émile Durkheim a esquissé, de fait, un programme de sociologie du curriculum attentif aux disciplines et à leurs évolutions, mais qui n'eut, en France tout au moins, qu'une postérité relative. Fortement mobilisée par la question de la reproduction des inégalités sociales au sein de l'école, la sociologie s'y est en effet montrée très attentive aux phénomènes de domination, en privilégiant la part d'arbitraire véhiculée par la culture scolaire et en n'entrant que peu, de ce fait, dans le teneur des disciplines. Néanmoins des travaux, comme ceux de Viviane Isambert-Jamati consacrés aux activités d'éveil à l'école élémentaire (Isambert-Jamati, 1984) ou à la pédagogie du Français au lycée (IsambertJamati et Grospiron, 1984), ou ceux de Tanguy (1983) sur les emplois du temps respectifs d'élèves de formations professionnelles et de formations générales, ré-ouvrirent la voie à des recherches contemporaines désireuses d'entrer davantage dans le processus de transmission de la culture par l'école. Les apports de la sociologie en matière de contextualisation des phénomènes de transmission aident, de ce point de vue, à " dénaturaliser » les disciplines en éclairant ce qui, en leur sein même, se prête ou non à la constitution de groupes d'acteurs qui tentent d'en imposer les versions qu'ils jugent légitimes. Pour ne s'en tenir qu'aux mathématiques, les travaux de Cooper (1997) aident à comprendre en quoi une discipline, quoiqu'unique dans sa dénomination, est de fait partagée par des orientations différentes et potentiellement concurrentes. Portées par des acteurs internes à la communauté scientifique, leurs variantes "pures » ou " appliquées » s'inscrivent différentiellement dans la demande sociale. Le développement de l'analyse numérique au détriment de l'algèbre structurale a pu ainsi affecter les programmes de l'enseignement secondaire britannique, en raison de l'intérêt qu'elle représentait pour la programmation linéaire elle-même appelée par le développement de l'informatique.

\section{Une migration conceptuelle}

Le dialogue entre sociologie et didactique est, d'une certaine manière, commencé du fait que l'une et l'autre intègrent soit la spécificité, soit la généralité des phénomènes contre laquelle elles se sont construites. Dans une des premières mises en dialogue de ces approches scientifiques, Johsua et Lahire (1999) mettent en évidence la prise en compte croissante, par chacune, de l'exigence secondaire pour l'une, mais première pour l'autre. La sociologie, elle-même plurielle comme toute science, s'est ainsi, notamment par le biais des travaux sur la réception culturelle, montrée de plus en plus sensible à la spécificité sémiotique des œuvres que son intérêt pour les fonctions sociales tendait à minimiser. La didactique pour sa part, initialement et centralement intéressée par leurs raisonnements généraux, donne de plus en plus « de chair » aux élèves en s'intéressant par exemple aux rapports aux sciences des filles et des garçons. Mais la question qui se pose désormais est de savoir si une singularisation des objets qui ne perde pas de vue les problèmes théoriques généraux, est seulement possible à partir des concepts de 
chacune de ces approches, ou si elle gagnerait à faire l'objet d'une problématisation commune. La notion de "contrat », travaillée par les deux disciplines de recherche fait par exemple l'objet, dans le dialogue entre Samuel Johsua et Bernard Lahire, d'une tentative de mise en commun d'analyses qui éclairent les succès ou les échecs d'apprentissage. Un colloque, tenu à Lausanne sur le thème ${ }^{1}$, a récemment mis l'accent sur la nécessité de mettre fin à la division du travail intellectuel sur ces questions. Prenant acte d'évolutions internes à la didactique et à la sociologie, il s'est demandé s'il était possible de dépasser leurs frontières disciplinaires pour construire de nouveaux concepts et les faire migrer d'un champ de recherche à l'autre.

\section{Un travail de problématisation}

\section{Le carrefour des apprentissages}

Sans viser une unité qui ne serait que factice, les contributions à la journée d'étude de l'ARCD mettent en évidence une volonté de faire communiquer des acceptions et des pratiques de la didactique, sans doute moins exclusives les unes des autres qu'il n'y paraît. Les risques sont néanmoins nombreux de ce que ce travail de rapprochement se fasse davantage pour satisfaire à des pressions institutionnelles (l'obtention de subventions par une discipline mieux reconnue, une meilleure reconnaissance universitaire, etc.) que pour développer un réel programme scientifique. Si l'idée de développer une didactique comparée se fraie un chemin, plusieurs des intervenants qui l'ont déjà adoptée, ou se disent prêts à le faire, font néanmoins remarquer la difficulté de la chose. Se posent en effet des questions redoutables, comme celle de savoir si un unique chercheur doit et peut couvrir les champs des diverses didactiques pour se livrer à de la comparaison. La création de chaires ou de postes de didactique comparée, qui semble une avancée dans la reconnaissance institutionnelle de la didactique peut notamment, selon la nature plus ou moins spécialisée ou transversale des profils choisis, fragmenter à nouveau un milieu de recherche au moment où elle cherche à le structurer. Les quelques propositions qui suivent visent à dépasser un tel dilemme, en faisant de la question des apprentissages des élèves un possible carrefour où s'articuleraient des versions complémentaires de la didactique, ainsi que des apports d'autres disciplines, au rang desquelles la sociologie.

La voie ouverte par Comenius (1684/2005), rappelée par Bernard Schneuwly lors de cette journée d'étude, est celle qui fait de la didactique une théorie de trois aspects très étroitement imbriqués: enseigner, apprendre et savoir. Un tel point de vue englobe en effet les caractéristiques liées aux conditions générales de la transmission, aux situations particulières dans lesquelles elle intervient, aux jeux d'acteurs qui la rendent ou non possible. Car l'épaisseur des situations d'apprentissage contraint à la prise en compte de ces trois dimensions de la transmission et de ses dispositifs et, de ce fait, à une reproblématisation (au sens de Jean-Louis Martinand) des connaissances antérieurement acquises dans une visée plus ou moins générique ou spécifique. Elle incite à dégager les caractéristiques de l'action conjointe entre enseignants et enseignés (Sensevy et Mercier, (Éds.) 2007), à dialectiser la notion de "discipline » dans son double statut de consistance théorique et de mise en œuvre hic et nunc (Reuter, 2007). La sociologie, lorsqu'elle accepte de payer le prix du détail et de s'intéresser au réel de l'activité d'enseignementapprentissage, dépasse elle-même ses propres cloisonnements en pointant les isomorphismes sous les différences de rôle, comme dans le cas des « routines incertaines » qui caractérisent aujourd'hui tant l'activité d'enseigner que celle d'apprendre (Barrère, 2003). Mais l'idée de l'unification d'un champ du « docere, discere, scire » relève vraisemblablement plus de l'idée directrice (Kant, 2006/1981) que du concept. De ce point de vue, la figure d'un didacticien gyrovague (au sens de Chevallard), sans affiliation disciplinaire connue, joue un rôle heuristique certain. Mais son incarnation dans tel ou tel individu, telle ou telle institution, replongerait dans les mêmes apories que celles qu'elle souhaite dépasser. De même, la construction d'une sociodidactique, comme discipline nouvelle, ni sociologique ni didactique, morcellerait vraisemblablement à nouveau un paysage qu'elle ambitionne d'unifier.

\section{Contrats didactiques différentiels et malentendus}

\section{Les contrats didactiques différentiels}

La notion de contrat didactique peut constituer une voie privilégiée pour la construction d'une espace 
commun de problématisation des situations d'apprentissage. Sa déclinaison en « contrat didactique différentiel » (Schubauer-Leoni, 1988) permet, par exemple, de considérer les interactions didactiques comme des cas particuliers d'interactions sociales et offre ainsi la possibilité d'un éclairage sociologique qui la contextualise. Travaillée dans le réseau Reseida ${ }^{2}$, elle permet de structurer les multiples enregistrements et observations réalisés dans les classes, qui révèlent des modes d'interaction récurrents entre maîtres et élèves faits d'attentes réciproques très différenciées concernant les « bons» élèves et les autres (Rochex, 2011). Des cadrages, plus ou moins faibles ou contraignants, des activités en classe peuvent ainsi, comme dans le cas d'une recherche d'antonymes de l'adjectif « léger », orienter les élèves vers une utilisation du dictionnaire très inégalement maîtrisée par eux et ne pas les aider à maîtriser le sens même de l'exercice. Faut-il chercher à « léger» ou à " repas » et aux autres mots de la liste proposée? Faut-il même se référer au dictionnaire ou vaut-il mieux mobiliser ses connaissances culturelles et lexicales? De même, des modalités différentes d'enrôlement, comme lors du passage au tableau, peuvent très bien aider à l'avancement du temps didactique, en mobilisant comme auxiliaires pédagogiques des élèves perçus comme de bon niveau ou à la mise en évidence de ce que d'autres élèves montrent d'exemples à ne pas suivre, en particulier en termes de comportement. D'autres séances montrent des reconfigurations à la baisse des tâches par lesquelles les maîtres finissent par faire à la place de certains élèves et les créditent d'une fausse réussite. Ces pratiques de classe participent vraisemblablement à la mise en place de tels contrats différentiels et, à terme, différenciateurs.

\section{Les malentendus socio-cognitifs et les registres de travail des élèves}

La notion de "malentendus socio-cognitifs » peut aider à analyser les conséquences de tels contrats, d'un point de vue qui mobilise simultanément la didactique et la sociologie. De tels malentendus, co-construits entre enseignants et élèves mais aussi avec d'autres acteurs, mélangent de manière inopportune les logiques cognitives spécifiques de l'école et des logiques sociales (Bautier \& Rochex, 2007, Bautier \& Rayou, 2013a). Ils consistent généralement en confusions sur la nature et les exigences du travail scolaire et organisent d'importants et persistants clivages entre élèves qui pensent surtout à s'acquitter de tâches, là où d'autres visent des apprentissages plus génériques et cumulatifs (Bonnéry, 2007). La prise en compte de plusieurs registres du travail des élèves (Bautier et Rayou, 2013b) tente d'approfondir la compréhension de tels malentendus et semble de nature à faciliter la collaboration des didacticiens et des sociologues en termes de re- et de co-problématisation.

Le registre proprement scolaire concerne les savoirs et les attitudes en jeu dans la classe. Il est lui-même composé de trois registres qui lui sont extérieurs et qu'il recompose, selon une logique propre en fonction des évolutions de ces derniers mais aussi d'une déclinaison qui dépend des degrés et des filières d'enseignement. L'un de ces registres, cognitif, relève des fonctions intellectuelles propres à l'humanité. Le second, culturel, est celui de valeurs liées à des savoirs généraux et à des pratiques qui excèdent la forme scolaire et nourrissent, de manière explicite ou implicite, les curriculums. Le troisième suppose l'appartenance à une communauté d'apprentissage, une identité symbolique légitimée par l'école dans laquelle doivent entrer les enfants et les jeunes pour devenir élèves. Face à un exercice donné, un élève doit se situer simultanément dans chacun de ces registres et en apprécier les exigences spécifiques pour y souscrire. Dans le cas de la « littératie étendue » qui suppose des usages du langage qui ne se réduisent pas à la narration d'expériences locales et spécifiques, l'élève idéal doit être capable d'opérations cognitives qui construisent comme texte les données, parfois éparses et hétérogènes, caractéristiques aujourd'hui de nombre d'exercices scolaires. Il doit, en même temps, comprendre et admettre que les savoirs et usages sociaux, auxquels se réfèrent de plus en plus les disciplines d'une école qui se veut ouverte sur la vie et la société, sont mobilisables à l'école mais au prix d'une transformation qui les rende compatibles avec la culture scolaire. Il doit enfin être capable de se situer comme un sujet, dont on sollicite l'engagement personnel mais médié par des règles d'objectivité qui signent l'appartenance à une cité savante.

La spécification de ces registres permet d'éclairer le système d'attentes à l'égard des élèves, d'un point de vue à la fois générique et spécifique, tant dans la synchronie des situations que dans la diachronie des cursus. Elle appelle des re-et co-reproblématisa- 
Patrick Rayou

tions, dans lesquelles la didactique n'est pas seulement convoquée pour ses apports en matière de transposition du cognitif en scolaire, mais se trouve requise comme indispensable pour comprendre en profondeur ce qui se joue aussi dans l'élaboration des valeurs culturelles et des identités symboliques à l'école. Les quelques considérations qui suivent tentent de proposer, à partir de travaux anciens ou actuels, des pistes pour une telle collaboration.

\section{Analyser les situations d'apprentissage}

La philosophie, discipline en quête de didactique

Mes activités de recherche, commencées avec des travaux sur la socialisation politique des lycéens (Rayou, 1998), se sont progressivement orientées vers la question des apprentissages dont la prise en compte me semble indispensable pour une sociologie de l'éducation désireuse d'entrer dans les « boîtes noires » de l'école. Rétrospectivement, ma recherche sur la dissertation de philosophie me montre ce qu'elle a dû à la problématisation didactique et ce qu'elle peut, en retour, lui apporter.

La confrontation du schéma prescrit de l'énonciation dans la dissertation (Cossutta, 1989; Raffin, 1994) et de sa version réelle dans les copies montre une fréquente renégociation du contrat didactique de la part des élèves. Le « je » universel attendu d'eux se transforme ainsi souvent en la juxtaposition de considérations qui n'impliquent aucun engagement de leur part; ils identifient le «tu » du destinataire au professeur ou au correcteur qu'il ne s'agit pas de convaincre mais de satisfaire; ils perçoivent et utilisent le «il » de l'intertextualité comme un réservoir éternel d'éléments constitutifs de la pensée philosophique. Ils transforment alors, par la substitution de la dispositio à l'inventio, l'activité délibérative attendue en démonstration d'une conclusion déjà élaborée (Delcambre, 1997).

Un tel retour à l'envoyeur de la responsabilité du problème (Rayou, 2005) ne peut se comprendre qu'à l'intersection des attentes didactiques d'une discipline et des mobilisations cognitives, culturelles et symboliques d'adolescents scolarisés dans un lycée massifié. Leurs manières de distinguer, à propos de sujets philosophiques, ce qu'il peut y avoir de passionnant dans les échanges entre pairs, d'intéressant dans les cours dans la discipline, mais d'effrayant dans la dissertation, permet de nourrir le programme de mise à jour de ce qu'est une conscience disciplinaire (Reuter, 2007).

Le cas de la philosophie semble particulièrement intéressant car cette discipline a résisté et résiste aux approches didactiques, au motif qu'elle serait à ellemême sa propre didactique. Elle justifie a fortiori la construction d'une espace commun de problématisation, s'appuyant tout autant sur son histoire institutionnelle et sur les catégories de l'expérience lycéenne contemporaine (Rayou, 2002), que sur les spécificités du philosopher (Tozzi, 1993) ou sur les ambiguités du traitement scolaire de la paraphrase (Daunay, 2002).

\section{Les arrière-plans des apprentissages}

Une autre recherche, conduite avec Gérard Sensevy, s'est intéressée à une activité d'enseignement-apprentissage en sciences de la vie et de la Terre, dans un temps et un lieu qui ne sont pas ceux de la classe au sens strict, mais de sa périphérie, l'aide aux devoirs (Rayou \& Sensevy, 2012). La lecture parallèle de cette séquence nous a conduits à des constatations proches sur la nature des apprentissages engagés et à rechercher, sinon un cadre théorique unique, du moins des notions et concepts qui semblaient convenir à l'une et l'autre discipline de recherche, tout en rendant compte de l'essentiel de l'objet étudié.

La séquence concerne une élève de $6^{\mathrm{e}}$ qui doit, à partir d'une fiche, faire plusieurs exercices relatifs à "L'unité du vivant: les cellules». Elle fait intervenir, outre l'élève, une assistante pédagogique, récemment recrutée, très bienveillante, mais peu armée par sa propre formation en matière de biologie. L'élève en question ne parvient à aucun moment à établir quelque rapport que ce soit avec le cours qu'elle a eu, en ignore les notions principales, attend passivement que l'assistante lui apporte des éléments de solution. Cette dernière, après quelques rappels méthodologiques généraux et des tentatives infructueuses de restaurer les notions importantes du cours, "valide» le travail fait par une élève docile heureuse de quitter le collège son devoir accompli.

Une telle scène, assez banale dans les collèges de l'éducation prioritaire, peut recevoir une explication en termes d' "arrière-plans » (Wittgenstein, 2004) sous-jacents aux transactions. D'un point de vue didactique, il faudrait, pour résoudre le problème, une équilibration entre contrat et milieu (Sensevy, 2011) qui ne peut survenir du fait de l'absence de 
connaissances partagées et pertinentes et de l'inadéquation de la fiche à la situation. D'un point de vue sociologique, cette même fiche constitue un dispositif qui, loin d'assurer la circulation d'un savoir entre classe et hors-classe, permet essentiellement aux deux protagonistes de se mettre en règle avec l'institution. Une exploration plus approfondie des arrière-plans montrerait, notamment, en quoi les présupposés de l'assistante pédagogique sur la possibilité de «voir » spontanément dans le bourgeonnement de la levure un signe qu'elle est un être vivant ou les prénotions de l'élève en matière de reproduction s'opposent à ce que la séance soit cognitivement féconde. Une contextualisation précise du dispositif de l'aide aux devoirs sur fond d'externalisation historique du travail des élèves contribuerait à éclairer en quoi ce type de sous-traitance (Kakpo, 2012) n'a que peu de chances de faire que les élèves qui le fréquentent progressent.

\section{CONCLUSION}

Une des difficultés majeures pour résoudre le problème, somme toute classique que représente, dans toute science, la dialectique du singulier et du pluriel de ses approches, provient peut-être de ce qu'elle est elle-même une discipline qui s'intéresse à des disciplines. Partageant avec elles le fait d'être, quel que soit son degré de reconnaissance, une institution, elle comprend une part d'institué qui tend à sa naturalisation. Se construire soi-même sur des disciplines dont on percevrait essentiellement l'aspect naturalisé risque de faire perdre de vue et la dynamique des disciplines d'enseignement étudiées et celle de la discipline universitaire qui se construit ce faisant.

Il semble donc nécessaire de sortir, au moins provisoirement, de la didactique organisée dans les différentes institutions qui la portent pour regarder ce qui, en elle comme dans toute activité de recherche, relève du singulier et du pluriel. Mais en distinguant ce qui est requis par des problématisations d'objets de ce qui relève d'unifications ou d'éclatements, liés aux rapports de force momentanés entre des formes disciplinaires qui peuvent correspondre à des espaces d'intéressement (Akrich, Callon \& Latour, 1991) sans recouper forcément des lignes de force épistémologiques.
Si l'on admet que le véritable point commun entre les démarches de recherche et celles d'enseignement est celui de la problématisation (Fabre, 1999), il peut être fécond d'organiser la réflexion sur la/les didactique(s) autour des apprentissages des élèves, où se joue en permanence la question de la généricité et de la spécificité des savoirs. Dans ce cas, la didactique peut rencontrer d'autres disciplines qui, comme la sociologie, ne se sont pas construites pour étudier les apprentissages, mais peuvent, notamment sur le thème du rapport à l'institution, l'aider à sortir d'elle-même pour mieux se retrouver.

\section{NOTES}

1. En référence à la Journée d'étude sur le thème « Didactique et/ou didactiques? D'une question polémique à la construction d'un espace de problématisation », organisé le 10 juin 2013, à Lyon, par l'ARCD et la Revue Education \& Didactique (Cf. Ligozat, Coquidé \& Sensevy, dans ce numéro).

2. Colloque Sociologie et didactique, Université de Lausanne, 13 et 14 septembre 2012.

3. REcherches sur la Socialisation, l'Enseignement, les Inégalités et les Différenciations dans les Apprentissages. 
Patrick Rayou

\section{RÉFÉRENCES}

Akrich, M., Callon, M. \& Latour, B. (1991). Lart de l'intéressement. In D. Vinck (coord.). Gestion de la recherche. Nouveaux problèmes, nouveaux outils, (p. 4-17). Bruxelles: De Boeck.

Barrère, A. (2003). Travailler à l'école. Que font les élèves et les enseignants du secondaire? Rennes: PUR.

Bautier, É \& Rochex, J.-Y. (2007). Apprendre: des malentendus qui font la différence. In J. Deauvieau. \& J.-P. Terrail (Éds). Les sociologues, l'école et la transmission des savoirs (p. 227-241). Paris: La Dispute.

Bautier, É. \& Rayou, P (2013a). Les inégalités d'apprentissage. Programmes, pratiques et malentendus scolaires. Paris: PUF.

Bautier, É. \& Rayou, P (2013b). La littératie scolaire: exigences et malentendus. Les registres de travail des élèves. Éducation et didactique, 7(2), 29-46.

Bonnéry, S. (2007). Comprendre l'échec scolaire. Paris: La Dispute.

Chervel A. (1988). LHistoire des disciplines scolaires. Réflexions sur un domaine de recherche. Histoire de l'éducation, 38(mai), 59-119.

Chervel A. (1977). Et il fallut apprendre à écrire à tous les petits Français: Histoire de la grammaire scolaire. Paris: Payot.

Chevallard Y. (1985/1991). La transposition didactique: du savoir savant au savoir enseigné. Grenoble: La Pensée Sauvage.

Comenius, J.A. (1684/2005). Novissima linguarum methodus. Genève: Droz.

Cooper, B. (1997). Comment expliquer les transformations dans les matières scolaires. In J.-C. Forquin. Les Sociologues de l'éducation américains et britanniques (p. 201-224). Bruxelles/Saint-Fons: De Boeck/INRP.

Cossutta, F. (1989). Éléments pour la lecture des textes philosophiques. Paris: Bordas.

Daunay, B. (2002). Éloge de la paraphrase. Saint-Denis: PUV.

Delcambre, I. (1997). L'exemplification dans les dissertations. Étude didactique des difficultés des élèves. Villeneuve d'Ascq: Septentrion.

Durkheim, É. (1990/1938), Lévolution pédagogique en France. Paris: PUF.

Fabre, M. (1999) Situations-problèmes et savoir scolaire. Paris : PUF.

Forquin, J.-C. (2008) Sociologie du curriculum. Rennes: PUR.

Isambert-Jamati, V. (1984). Culture technique et critique sociale à l'école élémentaire. Paris: PUF.

Isambert-Jamati, V. \& Grospiron M.-F. (1984). Types de pédagogie du français et différenciation sociale des résultats. L'exemple du « travail autonome» au deuxième cycle long. Études de linguistique appliquée, 54, 69-97 (repr. in Isambert-Jamati V. (1990) : Les savoirs scolaires, Paris: Editions Universitaires).

Kakpo, S. (2012). Les devoirs à la maison. Mobilisation et désorientation des familles populaires. Paris: PUF.
Kant, E. (2006/1781). Critique de la Raison pure. Paris: Flammarion.

Lahire B. \& Johsua S. (1999). Pour une didactique sociologique, Éducation et sociétés, 4, 29-56.

Martinand J.-L., (2001). Matrices disciplinaires et matrices curriculaires: le cas de l'éducation technologique en France. In C. Carpentier, (Coord.), Contenus d'enseignement dans un monde en mutation: permanences et ruptures (p. 249-269). Paris: LHarmattan.

Martinand, J.-L. (2006). Didactique et didactique. Esquisse problématique. In J. Beillerot et N. Mosconi (Dir.). Traité des sciences et pratiques de l'éducation ( $p$. 353367). Paris: Dunod.

Martinand, J/-L. (1986). Connaître et transformer la matière: des objectifs pour l'initiation aux sciences et techniques. Berne: Peter Lang.

Raffin, F. (Coord.) (1994). La dissertation philosophique. La didactique à l'œuvre. Paris: Hachette éducation.

Rayou, P. (2005). Contrat didactique et contrat social. La problématisation en philosophie. In M.-H. Salin, P. Clanché \& B. Sarrasy (Éds.). Sur la théorie des situations didactiques. Questions, réponses, ouvertures. (p. 359-355). Grenoble: La Pensée Sauvage.

Rayou, P. (2002). La «dissert de philo». Sociologie d'une épreuve scolaire. Rennes: PUR.

Rayou, P. (1998). La Cité des lycéens. Paris: L'Harmattan.

Rayou, P. \& Sensevy, G. (2012). Contrat didactique et contextes sociaux. La structure d'arrière-plans des apprentissages. Actes du Colloque Sociologie et didactique, Université de Lausanne, 13 et 14 septembre 2012. https://www.hepl.ch/files/live/sites/ systemsite/files/uer-agirs/Actualit \%C3\%A9s/Colloque $\% 20$ didactiques $\% 20$ et $\% 20$ sociologie/Contributions-du-colloque/atelier-3 \%E2 \%80 \%93savoirsdisciplinaires-et-rapport-au-savoir-des-enseignants/ milieux-didactiques-et-contextes-sociaux-les-arrieresplans-des-apprentissages-2012-uer-agirs-hep-vaud.pdf

Reuter, Y. (2007). La conscience disciplinaire. Présentation d'un concept, Éducation et didactique, 1(2), 57-71.

Rochex, J.-Y. (2011). Au cour de la classe, contrats didactiques différentiels et production d'inégalités. In J.-Y. Rochex \& J. Crinon (Dir.). La construction des inégalités scolaires. Rennes: PUR.

Schneuwly, B. (2011). Subject Didactics. An academic Field Related to the Teacher Profession ant Teacher Éducation. In B. Hubson, \& M. A. Meyer. Beyond Fragmentation: Didactics, Learning ant Teaching in Europe (p. 275-286). Opladen \& Farmington Hills (MI) : Barbara Budrich Publishers.

Schubauer-Leoni, M.-L. (1988). Le contrat didactique dans une approche psycho-sociale des situations d'enseignement. Communication au colloque « Le contrat didactique, différentes approches », Marseille (mars 1987). Interactions didactiques, 8, 63-75.

Sensevy, G. (2011). Le sens du savoir. Éléments pour une théorie de l'action conjointe en didactique. Bruxelles: De Boeck. 
Sensevy, G, \& Mercier, A. (Éds.), (2007). Agir ensemble. L'action didactique conjointe du professeur et des élèves. Rennes: PUR.

Tanguy, L. (1983). Savoirs et rapports sociaux dans l'enseignement secondaire en France. Revue française de sociologie, XXIV, 227-254.

Tozzi, M. (1993). Contribution à l'élaboration d'une didactique de l'apprentissage du philosopher. Revue française de pédagogie, 103,19-32.

Vincent, G., Lahire, B. et Thin D. (1994). L'Éducation prisonnière de la forme scolaire? Scolarisation et socialisation dans les sociétés industrielles. Lyon: PUL.

Wittgenstein, L. (2004). Recherches philosophiques. Paris: Gallimard. 